\title{
Role of microRNAs in chronic lymphocytic leukemia (Review)
}

\author{
XIANG-XIANG ZHOU $^{1}$ and XIN WANG ${ }^{1,2}$ \\ ${ }^{1}$ Department of Hematology, Provincial Hospital Affiliated to Shandong University; \\ ${ }^{2}$ Department of Diagnostics, School of Medicine, Shandong University, Jinan, Shandong 250021, P.R. China
}

Received March 1, 2013; Accepted July 19, 2013

DOI: $10.3892 / \mathrm{mmr} .2013 .1599$

\begin{abstract}
Chronic lymphocytic leukemia (CLL) is the most common type of leukemia among adults in the western world. It is characterized by a malignant clone of B cells in the bone marrow, blood and secondary lymphoid tissues. microRNAs (miRNAs) are a family of small, non-coding RNAs that regulate the expression of target messenger RNAs at the post-transcriptional level. Previous studies have suggested that miRNAs are extensively involved in the proliferation and differentiation of hematopoietic cells. Aberrant expression of certain miRNAs has been observed in CLL. Associations between miRNAs and chromosomal abnormalities suggest that miRNAs may be involved in the pathogenesis of CLL. Moreover, miRNAs may be used as novel biomarkers for the prognosis of CLL. Expression levels of miRNAs are also involved in resistance to chemotherapy drugs. In this article, we review recent developments of miRNAs in the initiation, prognosis and chemoresistance of CLL.
\end{abstract}

\section{Contents}

1. Introduction

2. miRNAs in the pathogenesis of CLL

3. miRNAs as hallmarks of CLL prognosis

4. miRNAs in CLL treatment and chemoresistance

5. Conclusion and future perspectives

\section{Introduction}

Chronic lymphocytic leukemia (CLL) is the most common type of leukemia among adults in the western world. The

Correspondence to: Professor Xin Wang, Department of Hematology, Provincial Hospital Affiliated to Shandong University, 324 Jingwu Road, Jinan, Shandong 250021, P.R. China

E-mail: xinwang55@yahoo.com.cn

Abbreviations: HSC, hematopoietic stem cell; GC B cell, germinal center B cell

Key words: microRNA, prognosis, chronic lymphocytic leukemia, pathogenesis incidence of CLL in the United States is $\sim 3.9 / 100,000$ and the median age of diagnosis is 72 (1). It is characterized by a malignant clone of $\mathrm{B}$ cells in the bone marrow, blood and secondary lymphoid tissues, which are primarily arrested in the G0/G1 cell-cycle phase. The clinical course of CLL is heterogeneous and survival times range from a few years to several decades. However, $~ 5-20 \%$ of CLL patients develop Richter's syndrome, an aggressive lymphoma which results in a short survival time (2).

microRNAs (miRNAs) are a family of small, evolutionarily conserved, single-stranded non-coding RNAs and are 18-25 nucleotides in length. The first miRNAs were discovered by Lee et al (3) in 1993. It has been demonstrated that miRNAs act as biological regulators involved in numerous cell processes, such as development, differentiation, proliferation, survival and death. They function by directly binding to the 3' untranslated regions (UTRs) of specific target messenger RNAs (mRNAs), leading to the inhibition of expression or degradation of target mRNAs. Recently, intensive studies on miRNA biogenesis and mechanisms by which target mRNAs are regulated by miRNAs have been conducted. A simple model of miRNA biosynthesis and function is shown in Fig. 1. miRNAs are encoded by genomic DNA in the nucleus and are transcribed by either RNA polymerase II (RNA Pol II) or III into primary miRNA transcripts (pri-miRNAs). pri-miRNA are processed by Drosha (also termed ribonuclease 3) and DiGeorge syndrome chromosomal region 8, and further cleaved into a hairpin-shaped 70-100 nucleotide precursor (pre-miRNA). Pre-miRNA is then actively transported into the cytoplasm by exportin 5 and processed by Dicer (another RNase III) and transactivating response RNA-binding protein into a 19-24 nucleotide, mature miRNA duplex by removal of the terminal loop. Generally, only one strand of the miRNA duplex is functional and the other strand is degraded. By associating with Argonaute proteins, mature miRNAs are incorporated into the RNA-induced silencing complex (RISC). miRNAs then guide the RISC to the 3'UTR of target mRNAs, leading to the inhibition of translation or the decrease in the stability of the target mRNAs $(4,5)$. Perfect or near perfect complementarity of miRNA with mRNA leads to the degradation of target mRNA and imperfect complementarity inhibits the translation of target mRNA (6). It has been demonstrated that miRNAs act as biomarkers in numerous types of cancer, such as breast and colorectal cancer (7). Furthermore, miRNAs are also important in almost every stage of hematopoietic cell development and signal transduction, which suggests the 


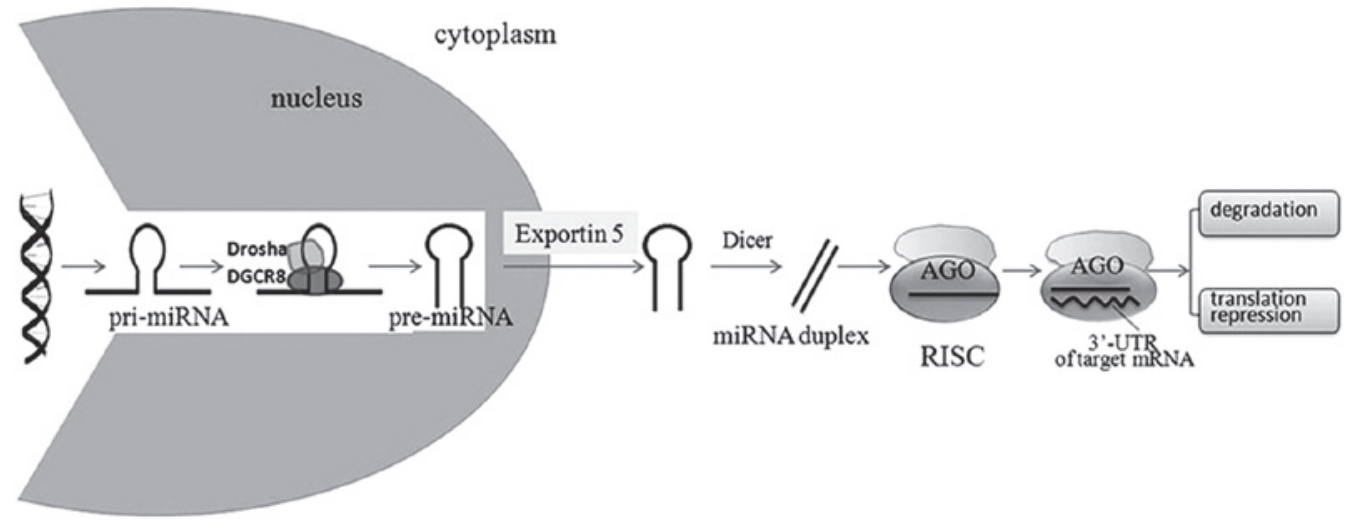

Figure 1. Biosynthesis and function of miRNAs. miRNA, microRNA.

potential significance of miRNAs in CLL, the most common type of leukemia in adults (8). In this review, we focus on previous studies which have investigated the involvement of miRNAs in the onset, prognosis and chemoresistance of CLL. Further understanding of miRNAs may provide novel diagnostic and therapeutic strategies for CLL.

\section{2. miRNAs in the pathogenesis of CLL}

miRNAs associated with the genetic aberration of CLL. Genetic aberrations are observed in $>80 \%$ of CLL patients (9). The most frequent alteration is del13q14, which accounts for $>50 \%$ of CLL cases. Calin et al (10) determined that a cluster of two miRNA genes, miR-15a and miR-16-1, were absent or downregulated in CLL patients with a $13 q$ deletion and this downregulation correlated with allelic loss at $13 q 14$. This was the first study to demonstrate that two miRNAs may act as suppressor genes of human cancer. A minimally deleted region containing deletions in the leukemia 2 (DLEU2) gene and the miR-15a/miR-16-1 genes has been identified in this region (10,11). However, Lia et al (12) determined that the size of the 13 q14 deletion was related to the penetrance and aggressiveness of lymphoproliferative diseases, which suggested another tumor suppressor function of genetic elements in addition to DLEU2/miR-15a/miR-16-1. Therefore, other genes are located in $13 \mathrm{q} 14$ as well as miR-15a and miR-16-1. miR-15a and miR-16-1 function as tumor suppressor genes through targeting several molecules involved in cell proliferation and apoptosis, such as B-cell CLL/lymphoma 2 (BCL2), cyclin D1 and D3 (CCND1 and CCND3) and cyclin-dependent kinase 6 (CDK6) $(13,14)$.

Another common aberration in CLL is trisomy 12 (10-20\%). It has previously been determined that NOTCH1 mutations were detected in approximately half of IGVH unmutated/ZAP70 ${ }^{+}$trisomy 12 CLL patients (41.9\%), which indicated that NOTCH1 activation is strongly correlated with trisomy 12 (15). However, López et al (16) suggested that the distribution of NOTCH1 mutations in CLL with trisomy 12 is heterogeneous and other chromosomal abnormalities including trisomy 18 altered the prognosis of these patients. Further studies are required to determine the correlation between NOTCH1 and trisomy 12 in CLL. The involvement of miRNAs in NOTCH1 mutations have been identified in $\mathrm{T}$ cell acute lymphocytic leukemia (T-ALL), including miR-181-a-1, miR-181-b-1 and miR-223 (17,18).

Deletion of 11q22-23 is observed in $20 \%$ of patients with advanced CLL (9) and predominantly impacts the ataxia-telangiectasia mutated (ATM) gene. Mutations of the ATM gene were detected in $12 \%$ of all CLL patients and $\sim 1 / 3$ of the CLL cases with del11q23 (19). ATM functions in regulating cell cycle progression and controlling the integrity of DNA repair. CLL patients with this deletion or mutation have a significantly shorter treatment-free interval (23.5 months) compared with those without such abnormalities (64.2 months) (20). A combination of an 11q deletion and an ATM mutation in CLL resulted in a shorter overall survival (OS) and progression-free survival (PFS) time following first-line therapy with alkylating agents and purine analogs (20). ATM is important in the regulation of miRNA biogenesis. It directly phosphorylates KH-type splicing regulatory protein (KSRP, a key component in Drosha and Dicer miRNA-processing complexes) upon DNA damage, facilitating the interaction between KSRP and pri-miRNAs and promoting miRNA maturation (21). Wang et al (22) demonstrated that miR-181 was directly targeted by ATM in breast cancer. Moreover, miR-34b and miR-34c are located in the 11 q23 region, thus deletion of this region led to the downregulation of these two miRNAs (23).

Deletion of $17 \mathrm{p} 13$ was observed in $\sim 5-10 \%$ of CLL cases at first diagnosis or treatment (9). Over $80 \%$ of CLL cases with $17 \mathrm{p}$ deletion carried TP53 (a tumor suppressor involved in cell cycling and cell death) mutations in the remaining allele. Inactivation of the second TP53 allele by point mutation was identified in numerous CLL cases and was correlated with chemorefractiveness and reduced survival (24). Expression of miR-34a, miR-29c and miR-17-5p were significantly downregulated in CLL with TP53 abnormalities (25).

miRNAs involved in B cell development. All blood cell lineages are generated from the hematopoietic stem cells (HSCs) in the bone marrow. miRNAs contribute to B cell development by targeting different transcription factors. Developmental arrest at the progenitor (pro) B cell to precursor (pre) B cell transition and an influence on antibody diversity is induced by knocking out Dicer in early B cells (26). The involvement of miRNAs during B cell development are shown in Fig. 2. Homeobox (HOX) family members, which are essential in modulating 


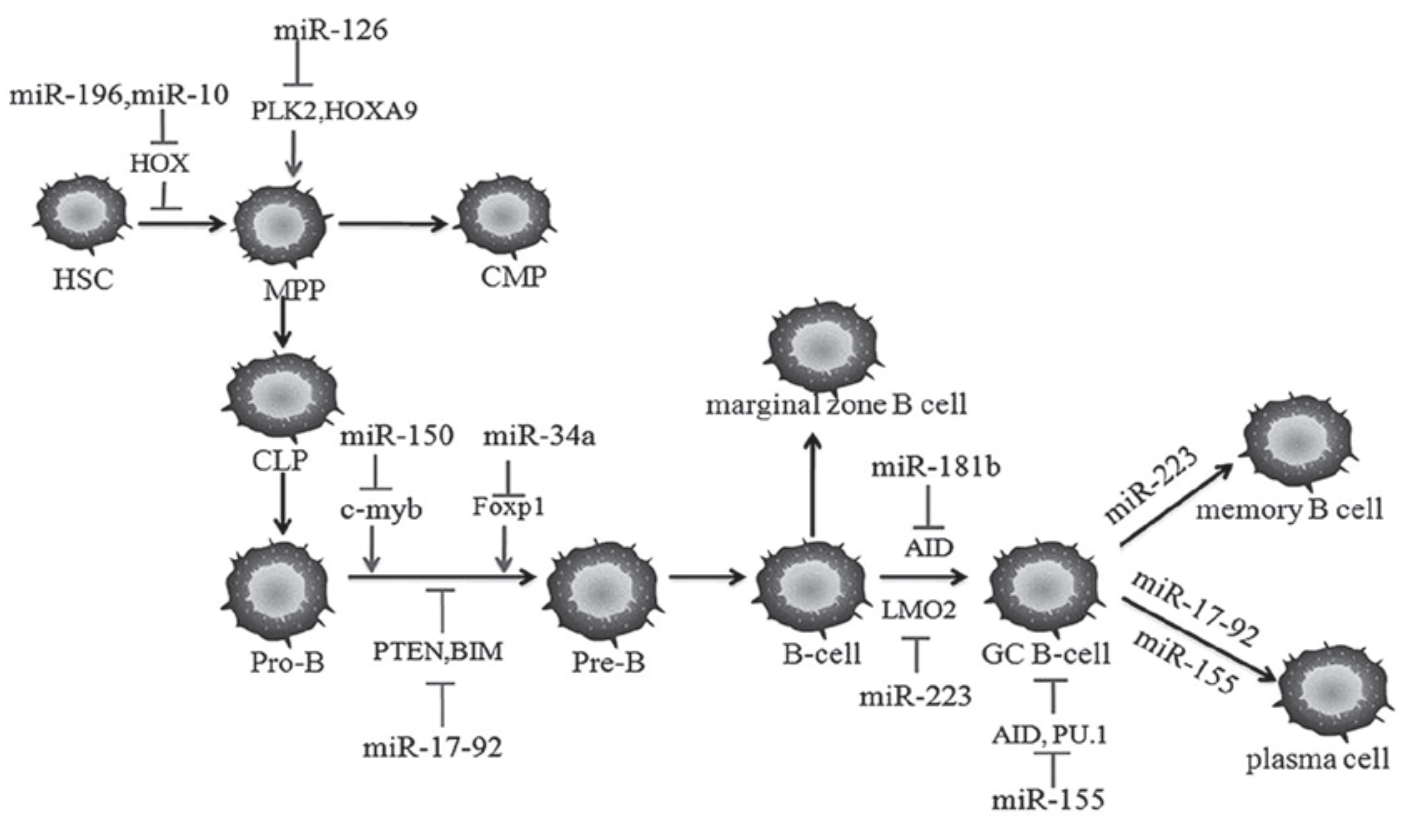

Figure 2. miRNAs involved in B cell development. miRNA, microRNA.

HSC homeostasis, are directly repressed by the miR-196b and miR-10 families, leading to the promotion of HSC differentiation. The development of progenitor cells is also blocked by miR-126, through the direct inhibition of the mRNAs encoding polo-like kinase 2 and HOXA9 (27). Constitutive expression of miR-150, a hematopoietic-specific miRNA, blocks the transition from pro-B cell to pre-B cell by inhibiting the expression of the translation factor, c-myb $(28,29)$. It has been demonstrated that the miR-17-92 cluster (miR-17-5p, miR-18a, miR-19a, miR-19b-1, miR-20a and miR-92) suppressed the expression of tumor suppressor phosphatase and tensin homolog (PTEN), which is often mutated in human lymphomas, and the pro-apoptosis protein BCL2-interacting mediator of cell death (30). This cluster is required for B cell development in normal hematopoiesis and increased expression of these genes was demonstrated to be oncogenic promoting the transition from pro-B cell to pre-B cell during B cell development (31). There are multiple putative binding sequences for miR-181b in the 3'UTR of activation-induced cytidine deaminase (AID) and these sequences are directly inhibited by miR-181b (32). miR-223 inhibits LMO2 in the transition from naïve B-cell to germinal center (GC) B-cell and GC B-cell to memory B-cell (33). Expression of miR-155 was observed to be at a moderate level in HSC, a high level in the germinal center and relatively lower level in mature B cells during normal lymphopoiesis (34). Upregulation of miR-155 has been observed in various human B-cell neoplasms, including diffuse large B cell lymphoma, Hodgkin's lymphoma and CLL. Numerous studies have demonstrated that miR-155 is important in the immune system and in homeostasis. Mice lacking miR-155 exhibited a normal steady condition of immune cell populations; however, showed a defective humoral response when immunized (35). miR-155 is upregulated subsequent to B cell activation in the GC, defective antibody class switching and reductive differentiation into plasma cells occurs in miR-155 deficient B cells (36). PU.1 and AID are two targets through which miR-155 functions. However, miR-155 and miR-181b do not exhibit overlapping functions in regulating AID expression as miR-181b prevents premature AID activity in the early stages of activation and miR-155 functions at a relatively later activation time. High levels of miR-34a also restricts the transition from pro-B to pre-B cell through inhibiting forkhead box transcription factor 1 (37).

miRNAs in the canonical Wnt signaling pathway. Numerous studies have demonstrated that the Wnt signaling pathway is significant in embryonic development and fundamental cellular functions throughout life. It has recently been demonstrated that the Wnt/ $\beta$-catenin signaling pathway is significant in tumorigenesis and angiogenesis (38). Constitutive activation of $\beta$-catenin inhibits multilineage differentiation from HSCs, including the development of B cells (39). Lu et al (40) observed the activation of the Wnt signaling pathway in CLL. Significantly higher expression levels of Wnt3, Wnt5b, Wnt6, Wnt10a, Wnt14, Wnt16, Fzd3 and LRP5/6, as well as LEF1 were observed in CLL cells compared with that in normal B cells. Survival of CLL lymphocytes was enhanced by SB-216763, an inhibitor of GSK3 $\beta$. Two small molecule inhibitors of the LEF-1/ $\beta$-catenin complex, CGP049090 and PKF115-584, were observed to efficiently induce the apoptosis of CLL cells in vitro and in vivo, whereas the normal B cells were not significantly affected (41). The Wnt canonical signaling pathway may be significant in the survival of CLL cells.

Several studies demonstrated the correlation between miRNAs and the canonical Wnt/ $\beta$-catenin signaling pathway. Valastyan et al (42) observed that Fzd3 was directly targeted by miR-31 in breast cancer and may have been a mechanism causing miR-31 to oppose metastasis. Analyses of the mRNA and protein levels determined that Wnt1 is transcriptionally inhibited by miR-21. Differentiation of monocyte-derived dendritic cells is inhibited by the antagonism of miR-21, either by adding exogenous Wnt1 or transfected with miR-21 
inhibitors (43). PTEN is an important tumor suppressor and the loss of PTEN results in the upregulation of anti-apoptotic factor myeloid cell leukemia 1 (MCL1) in HSC. PTEN was also shown to be targeted by miR-21 in hepatocellular cancer and epithelial ovarian cancer $(44,45)$. In addition, Rossi et al (46) suggested the 21FK (miR-21 expression, fluorescence in situ hybridization and karyotype) score, a three-category score model (scores of 0,1 or 2) to stratify the CLL patients according to OS. The lower the score, the longer the OS time in CLL patients. One point was allocated for miR-21 expression with a $17 \mathrm{p}$ deletion, and no points were allocated for low miR-21 expression or a normal karyotype. Patients with a score of zero exhibited a significantly higher survival rate than those with a score of 1 or 2. An miR-29a/Wnt positive feedback loop was discovered in osteoblastic differentiation. Transcription of miR-29 was induced by the canonical Wnt signaling pathway. Dikkopf-1, Kremen2 and secreted frizzled related protein 2, which are negative regulators of the canonical Wnt signaling pathway were directly inhibited by miR-29a. The expression levels of these Wnt signaling inhibitors were increased in miR-29a antagonist transfected cells (47). Furthermore, miR-315 and miR-135a/b directly targeted Axin and APC, respectively, in the canonical Wnt pathway (48). Few studies are available concerning miRNAs in the Wnt/ $\beta$-catenin signaling of CLL. miRNAs are involved in several components of the canonical Wnt signaling pathway; however, the majority of the specific mechanisms remain unknown possibly due to the crosstalk between Wnt/ $\beta$-catenin signaling and other pathways.

\section{3. miRNAs as hallmarks of CLL prognosis}

miRNAs in CLL with ZAP-70+ and/or unmutated $\operatorname{Ig} V_{H}$. Patients with aggressive CLL usually express the unmutated immunoglobulin heavy-chain variable-region gene $\left(\operatorname{IgV}_{\mathrm{H}}\right)$ and a high level of the 70-kDa $\zeta$-associated protein (ZAP-70), whereas patients with mutated $\mathrm{IgV}_{\mathrm{H}}$ and low ZAP-70 expression present with indolent CLL (49). Through an miRNA microarray composed of 190 human genes, Calin et al (50) identified a significant correlation between miRNAs and CLL with ZAP-70 expression and unmutated $\mathrm{IgV}_{\mathrm{H}}$. A signature of 13 miRNAs was identified, with upregulation of miR-15a, miR-16-1, miR-16-2, miR-23b, miR-24-1, miR-146, miR-155, miR-195 and miR-221, and downregulation of miR-223, miR-29a-2, miR-29b-2 and miR-29c in CLL with high ZAP-70 expression and unmutated $\mathrm{IgV}_{\mathrm{H}}$. Therefore, the expression of certain miRNAs may act as prognostic markers of CLL. The predictive value of circulating miRNAs in CLL disease stratification has been demonstrated (51). Expression levels of certain circulating miRNAs present in CLL patient plasma, such as miR-150 and miR-135, were significantly different from that in control groups. Circulating levels of certain miRNAs in CLL with ZAP-70 ${ }^{+}$were also different from those in ZAP-70- CLL patients. The level of miR-150 in the ZAP-70- CLL plasma was increased, which was thought to be correlated with the disease stage (51). Bomben et al (52) observed a significantly higher expression level of miR-17 (the prototypic member of the miR-17-92 family) in $\operatorname{IgV}_{\mathrm{H}}$ unmutated CLL cells with high ZAP-70 compared with that in CLL cells with mutated $\operatorname{IgV}_{\mathrm{H}}$ and a low ZAP-70 phenotype.
miRNAs and TP53 in CLL. The prognostic value of TP53 mutations in CLL has been shown in numerous studies. Zenz et al (53) demonstrated that the TP53 mutation acted as a central prognostic factor for PFS and OS in CLL independent of $17 \mathrm{p}$ deletions. TP53 mutation and/or 17p deletions were identified in $29 \%$ of CLL patients refractory to fludarabin in first-line treatment settings. Notably, the correlation between TP53 mutations and certain other factors, such as IGHV gene mutations, CD38 and ZAP-70 expression, or any chromosomal abnormality (other than 17p deletion) was of no significance (24). Therefore, TP53 mutations may be beneficial if added to the genetic tests for CLL. microRNA/TP53 feedback circuitry was demonstrated to be correlated with CLL pathogenesis and outcome (54). In this feedback circuit, TP53 acts as a molecular linker between $\mathrm{miR}-15 \mathrm{a} / \mathrm{miR}-16-1$ and $\mathrm{miR}-34 \mathrm{~b} / \mathrm{miR}-34 \mathrm{c}$ clusters, which are located on chromosome $13 \mathrm{q}$ and chromosome 11q, respectively. miR-15a and miR-16-1 directly bind to the 3'UTR of TP53 and inhibit its expression. TP53 binds directly to a TP53 binding site on pre-miR-34b/miR-34c to promote their translation and the downstream signal is activated. In CLL cases with a $13 \mathrm{q}$ deletion only, downregulated or absent miR-15a/miR-16-1 resulted in increased levels of BCL, MCL1 and TP53, further activated the expression of miR-34a, miR-34b and miR-34c and induced ZAP-70 inhibition. Thus, in CLL patients with a $13 q$ deletion, the number of apoptotic cells may be decreased due to the increased levels of anti-apoptotic proteins; however, the increase in tumor burden is relatively low due to the enhancement of the TP53 tumor suppressor pathway (54). This feedback circuitry provides a novel mechanism for the involvement of miRNAs in CLL with 13q deletions and their interaction with TP53. Mraz et al (55) demonstrated the involvement of miRNAs in cases of CLL with TP53 mutation. Thirty-five miRNAs were detected in CLL samples with TP53 mutations $(n=12)$ and wild type TP53 samples $(n=18)$, as a consequence, miR-34a, miR-29 and miR-17-5p were significantly downregulated in CLL with mutated TP53. miR-34a, which is located in $1 \mathrm{p} 36$ and is frequently deleted in numerous types of tumors, was the most differentially expressed miRNA with an 29 -fold decrease in CLL with mutated TP53. miR-29 was reported to directly downregulate the expression levels of TCL1 and MCL1 in CLL. As a member of the miR-17-92 family, miR-17-5-p directly targeted E2F1, p21 and cyclin D1, and other genes involved in the G1/S-phase cell-cycle transition.

miRNAs involved in Notch signaling. Leong and Karsan (56) observed the activation of NOTCH1 in leukemia through the study of the chromosomal translocation $\mathrm{t}(7 ; 9)(\mathrm{q} 34 ; \mathrm{q} 34.3)$ in $\mathrm{T}$ cell acute lymphoblastic cases (56). Activating mutations of NOTCH1 were observed in $8.3 \%$ of CLL at diagnosis, $31 \%$ in CLL transformation to Richter's syndrome and $20 \%$ in CLL chemorefraction (57). CLL patients with a NOTCH1 mutation presented with a more advanced clinical stage at diagnosis and a shorter 10-year OS (21\%) compared with those without NOTCH1 mutations (56\%) (58). NOTCH1 mutation in CLL generates a premature stop codon and leads to a constitutively activated and more stable form of the NOTCH1 protein, which lacks the C-terminal domain which contains a PEST sequence. Mutations of NOTCH1 act as an independent prognostic marker of CLL, which is prone to be mutually exclusive 
with TP53 mutations and a poor prognosis (59). Microarray profiling of miRNAs in T-ALL cells was performed prior to and following inhibition of Notch signaling with $\gamma$-secretase inhibitor (GSI). The expression level of miR-223 significantly increased $\sim 1$.5-fold when Notch signaling was inhibited by GSI. miR-223 targeted the 3'UTR of the insulin-like growth factor 1 receptor (IGF1R) and downregulated its expression. Thus, Notch signaling inhibits the miR-223 expression level in T-ALL cells and the total IGF1R protein levels are negatively regulated by miR-223 (18). miR-181a and miR-181-b-1, miRNAs related to NOTCH1, are also observed in T-ALL. Deletion of miR-181a-1/miR-181b-1 significantly inhibits the NOTCH1 oncogene-induced T-ALL by downregulating the negative regulators downstream of the NOTCH and pre-TCR signaling pathway (17). In addition, miR-181a/miR-181-b promoted the development of NK cells from CD34 ${ }^{+}$hematopoietic progenitor through the suppression of nemo-like kinase (NLK), a protein kinase that negatively regulates Notch signaling by inhibiting the formation of the Notch active transcriptional complex. Transcription levels of miR-181a/b increased during NK cell development from NK progenitor cells to final $\mathrm{CD}^{2} 6^{+} \mathrm{NK}$ cells. Moreover, miR-181a/miR-181b also increased IFN- $\gamma$ production in primary $\mathrm{CD}^{2} 6^{+} \mathrm{NK}$ cells, possibly by regulating the Notch signaling pathway (60). In addition, expression of miR-451 and miR-709 were repressed by intracellular NOTCH1 in a T-ALL mouse model through induction of the degradation of the E2A tumor suppressor (61).

In conclusion, the results of these studies suggest that miRNAs involved in the NOTCH1 mutation are important in several diseases, particularly T-ALL. A previous study demonstrated that NOTCH1 may be added to the hierarchical prognostic classification in CLL (62). However, few studies have been conducted concerning the correlation between miRNAs and NOTCH1 mutation in CLL at present. Thus further studies regarding the mechanism of miRNAs and NOTCH1 in CLL may aid in a more accurate prognosis for CLL patients.

\section{4. miRNAs in CLL treatment and chemoresistance}

Current therapy for the clinical treatment of CLL consists of chemotherapy with agents such as fludarabine, cyclophosphamide, bendamustine and chlorambucil, and immunotherapeutic agents such as rituximab and alemtuzumab (63). However, resistance to chemotherapeutic drugs is emerging as a challenging issue in the effective management of CLL patients. The p53 pathway has been demonstrated to be involved in the chemoresistance of CLL (64). Moreover, a model of the p53/miR-34a network exists in the DNA-damage response pathway in CLL, disturbance of this network results in chemoresistance. DNA damage leads to the induction of p53 through the activation of ATM. Arrest of the cell cycle and cell apoptosis are induced by $\mathrm{p} 53$ via direct targeting of p21, Puma, Rax and miR-34a. Therefore, a p53-dependent upregulation of miR-34a following DNA damage exists in CLL. An increase in miR-34a affects cell death through targeting CDK4, CDK6, CCND1, MYCN, BCL2 and Sirtuin 1. With TP53 mutation/17p deletion, ATM mutation/11q deletion or miR-34a downregulation, the DNA damage response pathway is reduced and the effect of cell apoptosis and cell cycle arrest is weakened resulting in chemoresistance (65). The expression of miR-34a is not only related to $17 \mathrm{p}$ deletion but also to fludarabine-refractory disease (in the absence of 17p deletion), mono-allelic TP53 mutation and an impaired DNA damage response. Expression of miR-34a is correlated with murine double minute 2 (MDM2) single nucleotide polymorphism 309 (SNP309) polymorphism (66). SNP309 leads to a T to G mutation in the first intron of MDM2 gene promoter and an enhancement in the MDM2 protein and further inhibits the function of p53 (67). Expression levels of miR-34a in CLL patients with SNP309 G/G genotype were significantly lower than in those with the TT genotype (66).

One of the most effective single therapeutic agents for CLL treatment is fludarabine. An investigation of miRNA expressions in fludarabine-sensitive and resistant cells demonstrated that miR-181a and miR-221 were significantly upregulated in resistant cases, and miR-29a was downregulated in resistant cases in vitro (68). Additionally, miR-181a and miR-181b significantly enhanced drug sensitivity in CLL cells by targeting the 3'UTR of BCL2, MCL1 and X-linked inhibitor of apoptosis protein (69). Ferracin et al (70) investigated the changes in the expression of 723 miRNAs in 17 CLL patients prior to and following 5-day fludarabine monotherapy. Differential expression of $37 \mathrm{miRNAs}$ were identified prior to and following fludarabine treatment. Among them, significant upregulation of miR-21, miR-222 and miR-148a were observed in non-responsive patients compared with patients sensitive to fludarabine treatment. Moreover, a significant increase in caspase activity of fludarabine-treated p53-mutant MEG-01 cells was induced by anti-miR-21 and anti-miR-222 oligonucleotides, suggesting the involvement of these two miRNAs in fludarabine resistance. These results indicated that miR-222 and miR-21 are involved in CLL fludarabine resistance independently of the p53 pathway. The studies demonstrated that miRNAs may be beneficial in the therapeutic approach to CLL by interfering with chemoresistance or as novel target therapy drugs. In the future, miRNAs may be used singly or coupled with other factors, or as target drugs.

\section{Conclusion and future perspectives}

In conclusion, miRNAs are extensively involved in the pathogenesis and the chemoresistant mechanisms of CLL, and may be used as prognostic markers. Cluster of miR-15a and miR-16-1 in CLL with del13q14 acts as a model of miRNA involvement in CLL pathogenesis. The development of the 21FK score suggests the beneficial implication of a single miRNA or an miRNA in combination with other prognostic markers in the accurate stratification of CLL. Due to the significant involvement of miRNAs in chemoresistance, novel therapeutic strategies and personalized therapy approaches may be developed. However, further studies are required to clarify the detailed mechanisms of specific miRNAs on their various targets and the association between miRNAs and bone marrow stromal cells prior to the use of miRNAs for clinical treatment of CLL.

\section{Acknowledgements}

This study was supported by the grants from the National Natural Science Foundation (grant no. 81270598), the 
Natural Science Foundation of Shandong Province (grant nos. Y2007C053 and 2009ZRB14176) and the Technology Development Project of Shandong Province (grant nos. 2007GG10 and 2010 GSF10250).

\section{References}

1. Dores GM, Anderson WF, Curtis RE, et al: Chronic lymphocytic leukaemia and small lymphocytic lymphoma: overview of the descriptive epidemiology. Br J Haematol 139: 809-819, 2007.

2. Tsimberidou AM and Keating MJ: Richter syndrome: biology, incidence, and therapeutic strategies. Cancer 103: 216-228, 2005

3. Lee RC, Feinbaum RL and Ambros V: The C. elegans heterochronic gene lin-4 encodes small RNAs with antisense complementarity to lin-14. Cell 75: 843-854, 1993.

4. Carthew RW and Sontheimer EJ: Origins and mechanisms of miRNAs and siRNAs. Cell 136: 642-655, 2009.

5. Kim VN, Han J and Siomi MC: Biogenesis of small RNAs in animals. Nat Rev Mol Cell Biol 10: 126-139, 2009.

6. Hutvágner G and Zamore PD: A microRNA in a multiple-turnover RNAi enzyme complex. Science 297: 2056-2060, 2002

7. Bartels CL and Tsongalis GJ: MicroRNAs: novel biomarkers for human cancer. Clin Chem 55: 623-631, 2009.

8. Joshi D, Gosh K and Vundinti BR: MicroRNAs in hematological malignancies: a novel approach to targeted therapy. Hematology 17: 170-175, 2012.

9. Dohner H, Stilgenbauer S, Benner A, et al: Genomic aberrations and survival in chronic lymphocytic leukemia. N Engl J Med 343: 1910-1916, 2000.

10. Calin GA, Dumitru CD, Shimizu M, et al: Frequent deletions and down-regulation of micro-RNA genes miR15 and miR16 at $13 q 14$ in chronic lymphocytic leukemia. Proc Natl Acad Sci USA 99: 15524-15529, 2002.

11. Lagos-Quintana M, Rauhut R, Lendeckel W and Tuschl T: Identification of novel genes coding for small expressed RNAs. Science 294: 853-858, 2001.

12. Lia M, Carette A, Tang H, et al: Functional dissection of the chromosome 13q14 tumor-suppressor locus using transgenic mouse lines. Blood 119: 2981-2990, 2012.

13. Cimmino A, Calin GA, Fabbri M, et al: miR-15 and miR-16 induce apoptosis by targeting BCL2. Proc Natl Acad Sci USA 102: 13944-13949, 2005.

14. Liu Q, Fu H, Sun F, et al: miR-16 family induces cell cycle arrest by regulating multiple cell cycle genes. Nucleic Acids Res 36 5391-5404, 2008.

15. Balatti V, Bottoni A, Palamarchuk A, et al: NOTCH1 mutations in CLL associated with trisomy 12. Blood 119: 329-331, 2012.

16. López C, Delgado J, Costa D, et al: Different distribution of NOTCH1 mutations in chronic lymphocytic leukemia with isolated trisomy 12 or associated with other chromosomal alterations. Genes Chromosomes Cancer 51: 881-889, 2012.

17. Fragoso R, Mao T, Wang S, et al: Modulating the strength and threshold of NOTCH oncogenic signals by mir-181a-1/b-1. PLoS Genet 8: e1002855, 2012.

18. Gusscott S, Kuchenbauer F, Humphries RK and Weng AP: Notch-mediated repression of miR-223 contributes to IGF1R regulation in T-ALL. Leuk Res 36: 905-911, 2012.

19. Austen B, Powell JE, Alvi A, et al: Mutations in the ATM gene lead to impaired overall and treatment-free survival that is independent of IGVH mutation status in patients with B-CLL. Blood 106: 3175-3182, 2005.

20. Skowronska A, Parker A, Ahmed G, et al: Biallelic ATM inactivation significantly reduces survival in patients treated on the United Kingdom Leukemia Research Fund Chronic Lymphocytic Leukemia 4 trial. J Clin Oncol 30: 4524-4532, 2012.

21. Zhang X, Wan G, Berger FG, He X and Lu X: The ATM kinase induces microRNA biogenesis in the DNA damage response. Mol Cell 41: 371-383, 2011.

22. Wang $\mathrm{Y}, \mathrm{Yu} \mathrm{Y}$, Tsuyada $\mathrm{A}$, et al: Transforming growth factor- $\beta$ regulates the sphere-initiating stem cell-like feature in breast cancer through miRNA-181 and ATM. Oncogene 30: 1470-1480, 2011.

23. Auer RL, Riaz S and Cotter FE: The 13q and 11q B-cell chronic lymphocytic leukaemia-associated regions derive from a common ancestral region in the zebrafish. Br J Haematol 137: 443-453, 2007.

24. Gonzalez D, Martinez P, Wade R, et al: Mutational status of the TP53 gene as a predictor of response and survival in patients with chronic lymphocytic leukemia: results from the LRF CLL4 trial. J Clin Oncol 29: 2223-2229, 2011.
25. Mraz M, Pospisilova S, Malinova K, Slapak I and Mayer J: MicroRNAs in chronic lymphocytic leukemia pathogenesis and disease subtypes. Leuk Lymphoma 50: 506-509, 2009.

26. Koralov SB, Muljo SA, Galler GR, et al: Dicer ablation affects antibody diversity and cell survival in the B lymphocyte lineage. Cell 132: 860-874, 2008.

27. O'Connell RM, Rao DS, Chaudhuri AA and Baltimore D: Physiological and pathological roles for microRNAs in the immune system. Nat Rev Immunol 10: 111-122, 2010.

28. Zhou B, Wang S, Mayr C, Bartel DP and Lodish HF: miR-150, a microRNA expressed in mature B and T cells, blocks early B cell development when expressed prematurely. Proc Natl Acad Sci USA104: 7080-7085, 2007.

29. Xiao C, Calado DP, Galler G, et al: MiR-150 controls B cell differentiation by targeting the transcription factor $\mathrm{c}-\mathrm{Myb}$. Cell 131: 146-159, 2007.

30. Xiao C, Srinivasan L, Calado DP, et al: Lymphoproliferative disease and autoimmunity in mice with increased miR-17-92 expression in lymphocytes. Nat Immunol 9: 405-414, 2008.

31. Ventura A, Young AG, Winslow MM, et al: Targeted deletion reveals essential and overlapping functions of the miR-17 through 92 family of miRNA clusters. Cell 132: 875-886, 2008.

32. de Yébenes VG, Belver L, Pisano DG, et al: miR-181b negatively regulates activation-induced cytidine deaminase in B cells. J Exp Med 205: 2199-2206, 2008.

33. Zhang J, Jima DD, Jacobs C, et al: Patterns of microRNA expression characterize stages of human B-cell differentiation. Blood 113: 4586-4594, 2009

34. Fernando TR, Rodriguez-Malave NI and Rao DS: MicroRNAs in B cell development and malignancy. J Hematol Oncol 5: 7, 2012.

35. Rodriguez A, Vigorito E, Clare S, et al: Requirement of bic/microRNA-155 for normal immune function. Science 316: 608-611, 2007.

36. Thai TH, Calado DP, Casola S, et al: Regulation of the germinal center response by microRNA-155. Science 316: 604-608, 2007.

37. Rao DS, O'Connell RM, Chaudhuri AA, Garcia-Flores Y, Geiger TL and Baltimore D: MicroRNA-34a perturbs B lymphocyte development by repressing the forkhead box transcription factor Foxp1. Immunity 33: 48-59, 2010

38. Ge X and Wang X: Role of Wnt canonical pathway in hematological malignancies. J Hematol Oncol 3: 33, 2010.

39. Seke Etet PF, Vecchio L and Nwabo Kamdje AH: Interactions between bone marrow stromal microenvironment and B-chronic lymphocytic leukemia cells: any role for Notch, Wnt and $\mathrm{Hh}$ signaling pathways? Cell Signal 24: 1433-1443, 2012.

40. Lu D, Zhao Y, Tawatao R, et al: Activation of the Wnt signaling pathway in chronic lymphocytic leukemia. Proc Natl Acad Sci USA 101: 3118-3123, 2004.

41. Gandhirajan RK, Staib PA, Minke K, et al: Small molecule inhibitors of Wnt/beta-catenin/lef-1 signaling induces apoptosis in chronic lymphocytic leukemia cells in vitro and in vivo. Neoplasia 12: 326-335, 2010.

42. Valastyan S, Reinhardt F, Benaich N, et al: A pleiotropically acting microRNA, miR-31, inhibits breast cancer metastasis. Cell 137: 1032-1046, 2009.

43. Hashimi ST, Fulcher JA, Chang MH, Gov L, Wang S and Lee B: MicroRNA profiling identifies miR-34a and miR-21 and their target genes JAG1 and WNT1 in the coordinate regulation of dendritic cell differentiation. Blood 114: 404-414, 2009.

44. Meng F, Henson R, Wehbe-Janek H, Ghoshal K, Jacob ST and Patel T: MicroRNA-21 regulates expression of the PTEN tumor suppressor gene in human hepatocellular cancer. Gastroenterology 133: 647-658, 2007.

45. Lou Y, Yang X, Wang F, Cui Z and Huang Y: MicroRNA-21 promotes the cell proliferation, invasion and migration abilities in ovarian epithelial carcinomas through inhibiting the expression of PTEN protein. Int J Mol Med 26: 819-827, 2010.

46. Rossi S, Shimizu M, Barbarotto E, et al: microRNA fingerprinting of CLL patients with chromosome 17p deletion identify a miR-21 score that stratifies early survival. Blood 116: 945-952, 2010.

47. Kapinas K, Kessler C, Ricks T, Gronowicz G and Delany AM: miR-29 modulates Wnt signaling in human osteoblasts through a positive feedback loop. J Biol Chem 285: 25221-25231, 2010.

48. Huang K, Zhang JX, Han L, You YP, Jiang T, Pu PY and Kang CS: MicroRNA roles in beta-catenin pathway. Mol Cancer 9: 252, 2010.

49. Rassenti LZ, Huynh L, Toy TL, et al: ZAP-70 compared with immunoglobulin heavy-chain gene mutation status as a predictor of disease progression in chronic lymphocytic leukemia. N Engl J Med 351: 893-901, 2004. 
50. Calin GA, Ferracin M, Cimmino A, et al: A MicroRNA signature associated with prognosis and progression in chronic lymphocytic leukemia. N Engl J Med 353: 1793-1801, 2005.

51. Moussay E, Wang K, Cho JH, et al: MicroRNA as biomarkers and regulators in B-cell chronic lymphocytic leukemia. Proc Natl Acad Sci USA 108: 6573-6578, 2011.

52. Bomben R, Gobessi S, Dal Bo M, et al: The miR-17 92 family regulates the response to Toll-like receptor 9 triggering of CLL cells with unmutated IGHV genes. Leukemia 26: 1584-1593, 2012.

53. Zenz T, Eichhorst B, Busch R, et al: TP53 mutation and survival in chronic lymphocytic leukemia. J Clin Oncol 28: 4473-4479, 2010.

54. Fabbri M, Bottoni A, Shimizu M, et al: Association of a microRNA/TP53 feedback circuitry with pathogenesis and outcome of B-cell chronic lymphocytic leukemia. JAMA 305: 59-67, 2011.

55. Mraz M, Malinova K, Kotaskova J, et al: miR-34a, miR-29c and miR-17-5p are downregulated in CLL patients with TP53 abnormalities. Leukemia 23: 1159-1163, 2009.

56. Leong KG and Karsan A: Recent insights into the role of Notch signaling in tumorigenesis. Blood 107: 2223-2233, 2006.

57. Fabbri G, Rasi S, Rossi D, et al: Analysis of the chronic lymphocytic leukemia coding genome: role of NOTCH1 mutational activation. J Exp Med 208: 1389-1401, 2011.

58. Puente XS, Pinyol M, Quesada V, et al: Whole-genome sequencing identifies recurrent mutations in chronic lymphocytic leukaemia. Nature 475: 101-105, 2011.

59. Rossi D, Rasi S, Fabbri G, et al: Mutations of NOTCH1 are an independent predictor of survival in chronic lymphocytic leukemia. Blood 119: 521-529, 2012

60. Cichocki F, Felices M, McCullar V, Presnell SR, Al-Attar A, Lutz CT and Miller JS: Cutting edge: microRNA-181 promotes human NK cell development by regulating Notch signaling. J Immunol 187: 6171-6175, 2011

61. Li X, Sanda T, Look AT, Novina CD and von Boehmer H: Repression of tumor suppressor miR-451 is essential for NOTCH1-induced oncogenesis in T-ALL. J Exp Med 208: 663-675, 2011
62. Mansouri L, Cahill N, Gunnarsson R, et al: NOTCH1 and SF3B1 mutations can be added to the hierarchical prognostic classification in chronic lymphocytic leukemia. Leukemia 27: 512-514, 2012.

63. Hallek M, Cheson BD, Catovsky D, et al: Guidelines for the diagnosis and treatment of chronic lymphocytic leukemia: a report from the International Workshop on Chronic Lymphocytic Leukemia updating the National Cancer Institute-Working Group 1996 guidelines. Blood 111: 5446-5456, 2008.

64. Zenz T, Mohr J, Edelmann J, et al: Treatment resistance in chronic lymphocytic leukemia: the role of the p53 pathway. Leuk Lymphoma 50: 510-513, 2009.

65. Zenz T, Mohr J, Eldering E, et al: miR-34a as part of the resistance network in chronic lymphocytic leukemia. Blood 113: 3801-3808, 2009.

66. Asslaber D, Piñón JD, Seyfried I, et al: microRNA-34a expression correlates with MDM2 SNP309 polymorphism and treatment-free survival in chronic lymphocytic leukemia. Blood 115: 4191-4197, 2010

67. Bond GL, Hu W, Bond EE, et al: A single nucleotide polymorphism in the MDM2 promoter attenuates the p53 tumor suppressor pathway and accelerates tumor formation in humans. Cell 119: 591-602, 2004

68. Moussay E, Palissot V, Vallar L, et al: Determination of genes and microRNAs involved in the resistance to fludarabine in vivo in chronic lymphocytic leukemia. Mol Cancer 9: 115, 2010.

69. Zhu DX, Zhu W, Fang C, et al: miR-181a/b significantly enhances drug sensitivity in chronic lymphocytic leukemia cells via targeting multiple anti-apoptosis genes. Carcinogenesis 33: 1294-1301, 2012.

70. Ferracin M, Zagatti B, Rizzotto L, et al: MicroRNAs involvement in fludarabine refractory chronic lymphocytic leukemia. Mol Cancer 9: 123, 2010. 